\title{
Matched-Filter Detection of Mode-Locked Laser Signals
}

\author{
R. J. D'Orazio and Nicholas George
}

\begin{abstract}
The passive Fabry-Perot cavity is shown to be a good practical approach to the match-filter optimization for the sensitive detection of mode-locked laser signals. Doppler measurements of relative motion over a wide range of velocities are possible simply by measuring the cavity length for a peak output.
\end{abstract}

\section{Introduction}

Multitone lasers of the mode-locked ${ }^{1}$ and cavitydumped $^{2}$ types emit their energy in short pulses. Sensitive detection of these emissions for point-topoint communications or echo-ranging systems can be accomplished by using appropriate filtering at the optical frequencies for detection and radio-frequency amplification. In the present work we describe our approach to matched-filtering for these signals.

In the literature related prior studies of laser detection include scanning Fabry-Perot cavities using a single pass-band of the passive cavity to analyze laser radiation ${ }^{3}$, spatial filtering techniques ${ }^{4}$, and various laser heterodyne techniques. ${ }^{5-7}$

Our optical receiver for mode-locked gas laser signals consists of a passive laser cavity controlled in length and a photodetector with its associated electronics. The length of the passive Fabry-Perot cavity is chosen approximately equal to the cavity length of the transmitting laser but with provision for fine fractional wavelength control of its length. In addition to the selective filtering characteristics of the passive cavity (passbands of unity transmission matching the frequencies of the multimode laser), a readout of the vernier length control, peaking the output, provides for an extremely wide range of velocity measurements with either an active or passive vehicle moving relative to the receiver.

\section{Passive Cavity: Matched-Filter for Mode- Locked Laser Radiation.}

Consider the passive cavity as shown in Fig. 1 where $h_{o}$ is the cavity length, $M_{1}$ is the fixed cavity mirror, and $M_{2}$ is the movable cavity mirror. The amplitude transmission function $T(\omega)$ may be shown to $\mathrm{be}^{8}$

R. J. D'Orazio is with Bell Laboratories, Holmdel, New Jersey 07733 , and N. George is with California Institute of Technology, Pasadena, California 91109.

Received 24 April 1972; revised 26 March 1973.

$$
T(\omega)=L \exp (i \psi(\omega)) /\left[1+P_{2} \sin ^{2}\left(\omega \frac{h_{0}}{c}\right)\right]^{1 / 2}
$$

where

$$
P_{2}=\left[4 r_{1} r_{2} \exp \left(-2 \alpha h_{0}\right)\right] /\left[1-r_{1} r_{2} \exp \left(-2 \alpha h_{0}\right)\right]^{2}
$$

and

$\psi(\omega)=-(\omega / c) h_{0}-\tan ^{-1}\left\{\frac{r_{1} r_{2} \sin \left[2 \omega\left(h_{0} / c\right)\right]}{1-r_{1} r_{2} \cos \left[2 \omega\left(h_{0} / c\right)\right]}\right\}$

and

$$
L=\left[t_{1} t_{2} \exp \left(-\alpha h_{0}\right)\right] /\left[1-r_{1} r_{2} \exp \left(-2 \alpha h_{0}\right)\right] .
$$

$t_{1}$ and $t_{2}$ are the transmission functions for mirror 1 and mirror 2 respectively, $r_{1}$ (mirror 1 right-side incidence) and $r_{2}$ (mirror 2 left-side incidence) are the reflection coefficients, $\alpha$ is the cavity loss per unit length, and $c$ is the speed of light.

To study the passive cavity as a multitone filter we use the matched-filter criterion resulting from the optimization of the SNR. So for a linear system with impulse response function $H_{1}(t)$ the total time varying output is $G_{1}(t)=H_{1}(t) \otimes\left[F_{1}(t)+N_{1}(t)\right]$ where $N_{1}(t)$ is the additive input signal and $\otimes$ indicates convolution. The matched-filter criterion specifies that the amplitude transmission function $H(\omega)_{m}=A F^{*}(\omega) / S_{n}(\omega)$ where $H(\omega)_{m}$ and $F(\omega)$ are the Fourier transform of $H_{1}(t)$ and $F_{1}(t)$ respectively, $S_{n}(\omega)$ is the power spectral density of the additive input noise, the asterisk denotes the complex conjugate, and $A$ is any nonzero complex constant. ${ }^{9}$ The subscript 1 will be used to denote time varying signals. Hence we see that the signal for which the passive cavity is a matched-filter is given by $F(\omega)=$ $S_{n}(\omega) T^{*}(\omega) / A^{*}$. Since the signals we shall be considering have a finite number of frequency peaks, we shall approximate $T(\omega)$ expanding around the zeroes, $\omega_{p}$, of $\sin ^{2}\left(\omega h_{o} / c\right)$ for a finite number of peaks around $\omega_{o}$ so that from Eq. (1) dropping $\psi(\omega)$ we have

$$
T(\omega)_{A}=L /\left[1+\left(\frac{2 \Delta \omega}{\Delta \omega_{p}}\right)^{2}\right]^{1 / 2}
$$

where $\Delta \omega=\omega-\omega_{p}=\omega-\left(\omega_{o}+p \omega_{c o}\right), \omega_{c o}=\pi c / h_{o}$ and $\Delta \omega_{p}=2 c /\left[h_{o}\left(\mathrm{P}_{2}\right)^{1 / 2}\right]$. We note that $\Delta \omega_{p}$ is the 


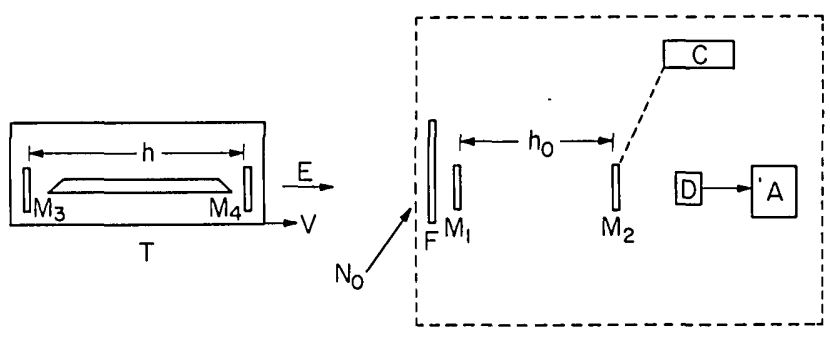

Fig. 1. Passive cavity receiver: $T$ laser transmitter; $M_{3}, M_{4}$ laser mirrors; $h$ laser cavity length; $E$ signal; $V$ velocity of laser relative to receiver; $N_{o}$ noise; $F$ coarse bandpass filter; $M_{1}, M_{2}$ passive cavity mirrors; $h_{o}$ passive cavity length; $D$ detector; $A$ detector electronics; $C$ mirror control.

full width at half power of each Lorentzian lineshape function generated by Eq. (5). Generally we shall assume white noise so that $S_{n}(\omega)=N_{o}$ is uniform over the frequencies of interest. Similarly we shall assume $t_{1}, t_{2}, r_{1}, r_{2}$ are constant over the frequencies of interest. Thus from Eq. (5) we see that the signal for which the passive cavity is a matched-filter is

$$
F(\omega)=\left[S_{n}(\omega) L\right] / A^{*} \sum_{p=N}^{N} 1 /\left[1+\left(\frac{2 \Delta \omega}{\Delta \omega_{p}}\right)^{2}\right]^{1 / 2} .
$$

Now for convenience we shall assume the idealized electric field amplitude at the output of an unmodulated mode-locked laser with $2 N+1$ modes is given by

$$
\begin{aligned}
E_{1}(t)= & \sum_{p=-N}^{N} \exp \left[i\left(\omega_{0}+p \omega_{c}\right) t\right] \\
& =\exp \left(i \omega_{0} t\right)\left\{\sin \left[(2 N+1) \frac{\omega_{c} t}{2}\right] / \sin \left(\frac{\omega_{c} t}{2}\right)\right\}
\end{aligned}
$$

where $\omega_{0}=n \pi c / h$ is the center frequency of the laser, $n$ is some large integer, $\omega_{c}=\pi c / h$ is the free spectral range, and $h$ is the effective cavity length. Then the Fourier transform of Eq. (7) is

$$
E(\omega)=2 \pi \sum_{p=-N}^{N} \delta\left[\omega-\left(\omega_{0}+p \omega_{c}\right)\right]
$$

where $\delta(\omega)$ is the Dirac delta function. Thus we see that $T(\omega)_{A}$ is a comb-filter for $E(\omega)$ for $h=h_{o}$, i.e., $T(\omega)_{A}$ has passbands of unity transmission matching the frequencies of the multimode laser.

For an actual laser signal, writing $E(\omega)$ for a multitone laser with finite linewidth will yield an expression as an alternative to the monochromatic idealization of Eq. (8). We note that the spacing between the tones of a mode-locked laser are determined by free spectral range, $\omega_{c}=\pi c / h$, of the laser cavity. It is our contention that the passive cavity transmission function will control the lineshape of the laser output if the gain $\alpha$ and the dispersion in the cavity are independent of frequency around a resonant peak. One may further consider this observation by noting that for $\mathrm{He}-\mathrm{Ne}$ the width of the Lorentzian shaped hole that is burned into the Doppler broadened gain profile at saturation is much broader than the mode-width of the laser cavity. Thus for $\alpha<0$ the frequency variation of $P$, Eq. (2), and $L$, Eq. (4), are negligible around the resonance.

In the literature related prior studies of the laser lineshape include lumped element LGC circuit models. ${ }^{10,11}$ Freed and Haus ${ }^{12}$ used the solution of the nonlinear Van der Pol oscillator equation to describe the spectrum of the laser output. The basic result of using a lumped circuit model is that the lineshape function is Lorentzian. ${ }^{13}$ The interesting point is that if one started with the transmission function for a cavity or a transmission line, the lineshape would be controlled by equations similar to Eq. (1).

Note that both the lumped circuit and passive cavity approaches to the laser spectrum assume that the random cavity mirror vibrations and effective cavity length fluctuations are negligible. If one dropped these assumptions the lineshape could be considered Gaussian or some other lineshape function.

So as the gain curve saturates, the right-half-plane poles of Eq. (1), for $s=i \omega$, will migrate to the $i \omega$ axis. We select the value of $\alpha$ which is an amount $\epsilon$ from the saturated pole so that Eq. (2) becomes

$$
P_{1}=\left[4 \exp \left(-2 \epsilon h_{0}\right)\right] /\left\{1-\left[\exp \left(-2 \epsilon h_{0}\right)\right]^{2}\right\} .
$$

Then we may write the spectrum of the laser signal with finite linewidths as

$$
E(\omega)=t_{1} t_{2} /\left[2\left(r_{1} r_{2}\right)^{1 / 2}\right] V / P_{1}\left\{1 /\left[1+P_{1} \sin ^{2} \omega\left(h_{0} / c\right)\right]^{1 / 2}\right\} .
$$

We note that a very good approximation to Eq. (10) may be obtained by expanding around the zeroes $\omega_{p}$ of $\sin ^{2} \omega\left(h_{o} / c\right)$ for $2 N+1$ tones analogous to the approximation of Eq. (5) so that the alternative to Eq. (8) becomes

$$
E(\omega)=\left(t_{1} t_{2}\right) /\left[2\left(r_{1} r_{2}\right)^{1 / 2}\right] V / P_{1} \sum_{p=-N}^{N} 1 /\left[1+\left(\frac{2 \Delta \omega}{\Delta \omega_{1}}\right)^{2}\right]^{1 / 2}
$$

where $\Delta \omega=\omega-\omega_{p}=\omega-\left(\omega_{o}+p \omega_{c}\right), \Delta \omega_{1}=2 c /$ $\left(h \vee P_{1}\right)$. Thus comparing Eq. (6) with Eq. (11) indicates that for $\Delta \omega_{p}=\Delta \omega_{1}$ and $h=h_{o}$ the passive cavity of Eq. (1) is a matched-filter for the multitoned signal of Eq. (10).

\section{Signal-to-Noise Ratio}

We compute the predetection time varying SNR at the output of the passive cavity as

$$
\operatorname{SNR}(t)=\left|T_{1} \bigotimes E_{1}\right|^{2} / R_{h n}(0)
$$

where $E_{1}$ is the inverse Fourier transform of Eq. (10) and $T_{1}$ is the inverse Fourier transform of Eq. (1) and $R_{h n}(0)$ is the auto correlation function evaluated at zero, which is equivalent to the mean-squared value of the additive noise at the output of the passive cavity given by

$$
R_{h n}(0)=\frac{1}{2 \pi} \int_{-\infty}^{\infty} S_{n}(\omega)|T(\omega)|^{2} d \omega .
$$

The numerator of Eq. (12) may be expressed by the inverse Fourier transform as 


$$
\left|T_{1} \bigotimes E_{1}\right|^{2}=\left.\left|\frac{1}{2 \pi} \int_{-\infty}^{\infty} T(\omega) E(\omega) \exp (i \omega t) d \omega\right|\right|^{2}
$$

and substituting Eq. (1) and Eq. (10) we may write the modulus-squared value of the signal portion of the output of a passive cavity as

$$
\begin{aligned}
& \left|T_{1} \otimes E_{1}\right|^{2}=\mid \frac{L t_{1} t_{2}}{2\left(r_{1} r_{2}\right)^{1 / 2}} / P_{1} \int_{-\infty}^{\infty} . \\
& \times \frac{\exp (i \omega t) d \omega}{\left[1+P_{1} \sin ^{2} \omega\left(h_{0} / c\right)\right]^{1 / 2}\left[1+P_{2} \sin ^{2} \omega\left(h_{0} / c\right)\right]^{1 / 2}} \mid .
\end{aligned}
$$

Since the signal has only $q=2 N+1$ modes we may write Eq. (15), with the substitutions $\omega=\omega_{0}-(c /$ $\left.h_{o}\right) x, t=h_{o} / c, \omega_{o}=(n \pi c) / h_{o}$, where $n$ is some larger interger, $\exp (-i x)=\cos x-i \sin x$ and noting that the sine integral over symmetric limits vanishes, as

$$
\left|T_{1} \otimes E_{1}\right|^{2}=(q / 2 \pi)^{2} L^{2}\left[\frac{t_{1} t_{2}}{2\left(r_{1} r_{2}\right)^{1 / 2}}\right]^{2}\left(2 c / h_{0}\right)^{2} K^{2}(m)
$$

where for $1 / P_{2}>1 / P_{1}>0, K(m)$ is the complete elliptic integral of the second kind given by

$$
K(m)=\int_{0}^{\pi / 2}\left[(d \theta) /\left(1-m^{2} \sin ^{2} \theta\right)^{1 / 2}\right]
$$

and

$$
m=\left[\left(1 / P_{2}\right)-\left(1 / P_{1}\right)\right]^{1 / 2} /\left(1 / P_{2}\right)^{1 / 2} .
$$

The denominator of Eq. (12) may be expressed from Eq. (1) and Eq. (13) as

$$
\begin{aligned}
R_{h n}(0)=\frac{L^{2}}{2 \pi} \int_{q\left[(\pi c) / h_{0}\right]} \frac{S_{n}(\omega) d \omega}{1+} P_{2} \sin ^{2}\left[\omega\left(h_{0} / c\right)\right] \\
=\frac{L^{2} N_{0}}{2 \pi} q \frac{\pi c}{h_{0}} \frac{1}{\left(1+P_{2}\right)^{1 / 2}}
\end{aligned}
$$

where, as in the integration above, $q$ is the number of free spectral ranges over which the integration is taken. Thus the peak SNR for the passive cavity taking the ratio of Eq. (16) to Eq. (17) is given by

$$
\begin{array}{r}
\operatorname{SNR}_{p}=4(q / 2 \pi)\left[t_{1} t_{2} / 2\left(r_{1} r_{2}\right)^{1 / 2}\right]^{2}\left(c / h_{0}\right)\left(1+P_{2}\right)^{1 / 2} K^{2}(m) \\
/\left(\pi N_{0}\right) .
\end{array}
$$

In a similar fashion, expressing $E \bigcirc E^{*}$, and $R_{h n}(0)$ for the matched-filter to Eq. (10) and using the same assumptions as above, the peak SNR for the matched-filter is

$$
\begin{array}{r}
\left.\mathrm{SNR}_{1}=4(q / 2 \pi)\left[\left(t_{1} t_{2}\right) /\left[2\left(r_{1} r_{2}\right)^{1 / 2}\right]\right]\right]^{2}\left(c / h_{0}\right)\left[\tan ^{-1}\left(V P_{1}\right)\right]^{2} \\
\times\left(1+P_{1}\right)^{1 / 2} /\left(\pi N_{0}\right) .
\end{array}
$$

Thus the departure of the passive cavity from the matched-filter is from Eqs. (18) and (19)

$$
\begin{aligned}
\mathrm{SNR}_{p} / \mathrm{SNR}_{1}=\left(1+P_{2}\right)^{1 / 2} /\left(1+P_{1}\right)^{1 / 2} \cdot\left[K^{2}(m)\right. \\
/\left[\tan ^{-1}\left(V P_{1}\right)\right]^{2}
\end{aligned}
$$

Since $\left.P_{1} \gg 1, \Delta \omega_{p}=2 c / h_{o} \sqrt{ } P_{2}\right)$, and $\Delta \omega_{1}=2 c /$ $\left(h_{o} \sqrt{ } P_{1}\right)$ then

$$
\mathrm{SNR}_{p} / \mathrm{SNR}_{1}=(2 / \pi)^{2}\left(\Delta \omega_{1} / \Delta \omega_{p}\right) K^{2}(m) .
$$

Hence the SNR of the passive cavity approaches that of the matched-filter when $\Delta \omega_{l} \cong \Delta \omega_{p}$ as may be seen in the plot of Eq. (21) in Fig. 2.
The Fabry-Perot cavity is probably as close a physical realization to a matched-filter for the multitoned laser as can be attained in a passive system. Even so, gain narrowing invariably results in $\Delta \omega_{l}<$ $\Delta \omega_{p}$, thereby limiting the observed improvement in SNR from its optimal value. ${ }^{14}$ For high gain lasers with cavities of low finesse, the receiver can be made closer to ideal, while greater departures are to be expected in the case of low gain. We note, too, that larger bandwidths, $\Delta \omega_{p}$, are called for with information modulated lasers and cavity-dumped lasers where mode-locking may not have been employed.

To obtain the predetection SNR improvement with use of the passive cavity we note the SNR without the cavity is given by the ratio of the modulussquared value of Eq. (7) to Eq. (17) with $P_{2}=0$. Thus the SNR improvement with use of the passive cavity with respect to no cavity is given by Eqs. (1), (7), (12), (14), and (17) as

$$
\mathrm{SNR}_{\text {with }} / \mathrm{SNR}_{\text {without }}=\left(1+P_{2}\right)^{1 / 2} \text {. }
$$

Since the signal portion of the time varying output of the passive cavity for a mode-locked laser input is given by the real part of $T_{1} \otimes E_{1}$, from Eq. (1) and Eq. (8) dropping $\psi(\omega)$ we have

$$
G_{1}(t)=\sum_{p=-N}^{N}[\cos (a-b)] /\left(1+P_{2} \sin ^{2} b\right)^{1 / 2}
$$

where $a=\dot{*}\left\{\omega_{o}+[\pi c / h]\right\} t$ and $b=\left(h_{o} / c\right)\left\{\omega_{o}+\right.$ $[(p \pi c) / h]\}$, then we see the peak SNR occurring for $h$ $=h_{o}$. The SNR as a function of time and a function of relative cavity length $h_{o} / h$ is illustrated in Fig. 3 . The number of modes oscillating is 9 with a peak SNR of 49 and a period of 8 nsec. The increment of relative cavity length is $\Delta h_{o} / h=0.0001$.

\section{Rise-Time of the Passive Cavity}

In consideration of the rise-time, $\tau$, of the FabryPerot resonant cavity we start with the Laplace transform representation of the amplitude transmission function given by setting $s=i \omega$ in Eq. (1) we obtain

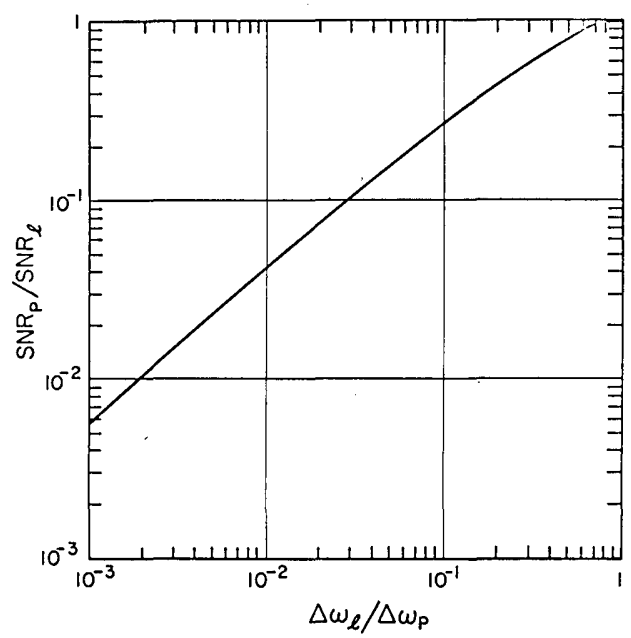

Fig. 2. Departure from matched-filter vs relative line widths. 


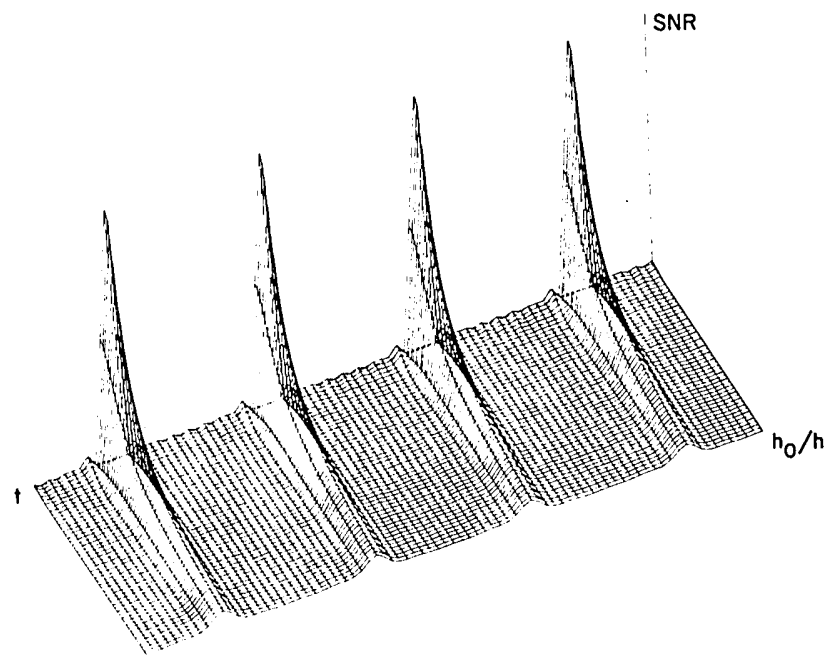

Fig. 3. Signal-to-noise as a function of time and relative cavity length.

$$
\begin{aligned}
& T(s)=\exp (-a s) T^{\prime}(s)=\left[T_{\alpha} \exp (-a s)\right] /[1 \\
& \text { - } \left.R_{\alpha} \exp (-2 a s)\right]
\end{aligned}
$$

where $\mathrm{T}_{\alpha}=t_{1} t_{2} \exp \left(-\alpha h_{o}\right), R_{\alpha}=r_{1} r_{2} \exp \left(-2 \alpha h_{o}\right)$, $a=\left(h_{o} / c\right)$. If we expand $T^{\prime}(s)$ in partial fractions and group the complex conjugate poles we obtain

$$
T^{\prime}(s)=\frac{T_{\alpha}}{a} \sum_{m=0}^{\infty}(s-\sigma) /\left[(s-\sigma)^{2}+\omega_{m}^{2}\right]
$$

where $\alpha=1 /(2 a) \ln R_{\alpha}$ and $\omega_{m}=m \pi c / h_{o}$. Now the Laplace transform of the real part of Eq. (7) becomes

$$
E(s)=\mathrm{L}\left[\operatorname{Real} E_{1}(t)\right]=\sum_{p=-N}^{N}\left[s /\left(s^{2}+\omega_{p}^{2}\right)\right] .
$$

So the signal output of the passive cavity for the idealized mode-locked laser input is given by

$$
G(s)=\exp (-a s) G^{\prime}(s)=\exp (-a s) E(s) T^{\prime}(s)
$$

where we note that $\exp (-a s)$ for a non-negative real constant corresponds to a time shift in the time domain. The inverse Laplace transform of a general term of $G^{\prime}(s)$ is given by

$$
\begin{aligned}
\mathrm{L}^{-1}\left[\frac{\dot{s}}{s^{2}+\omega_{p}^{2}} \cdot \frac{(s-\sigma)}{(s-\sigma)^{2}+\omega_{m}^{2}}\right] \\
\quad=\left[\frac{1+\sigma^{2}}{\left(\sigma^{2}+\omega_{m}^{2}-\omega_{p}^{2}\right)^{2}+4 \sigma^{2} \omega_{p}^{2}}\right]^{1 / 2} \\
\quad \times\left[\omega_{p} \sin \left(\omega_{p} t+\psi_{1}\right)+\omega_{m} \sin \left(\omega_{m} t+\psi_{2}\right)\right] .
\end{aligned}
$$

Thus for $\omega_{p}=\omega_{m} \gg \sigma, \sigma \gg 1$ the fraction of the maximum steady state output which is obtained in $\tau$ seconds is given by Eq. (27) and Eq. (28) as

$$
\begin{aligned}
f=1-\exp [\sigma(\tau-a)] u & (\tau-a) \\
& =\left(1-R_{\alpha}{ }^{(\tau-a) / 2 a}\right) u(\tau-a)
\end{aligned}
$$

where

$$
u(x)=\left\{\begin{array}{lll}
1 & x & >0 \\
0 & x & <0
\end{array} .\right.
$$

The $\omega_{p} \neq \omega_{m}$ terms are neglected in that the coefficients of the sine terms of Eq. (28) are on the order of $\ln R_{\alpha}$ which are negligible for $R_{\alpha} \approx 1$. Equation (29) is plotted in Fig. 4 as a function of time and number of pulse train bounces parameterized by $R_{\alpha}$ $=r_{1} r_{2} \exp (-2 \alpha h)$.

Thus the rise-time of the passive cavity is given by

$$
\tau=-1 / \sigma=-2 a\left[1 /\left(\ln R_{\alpha}\right)\right] \simeq 2\left(h_{0} / c\right) R_{\alpha} /\left(1-R_{\alpha}\right)
$$

and from Eq. (2)

$$
\tau=\left(h_{0} / c\right) \sqrt{ } P \sqrt{ } R_{\alpha}=\left(2 \sqrt{ } R_{\alpha}\right) /\left(\Delta \omega_{p}\right)=\sqrt{ } R_{\alpha} /\left(\pi \Delta \nu_{p}\right)
$$

and thus the rise-time-bandwidth product is

$$
\tau \Delta \nu_{p}=V R_{\alpha} / \pi \text {. }
$$

Where $R_{\alpha}=0.991, \tau \Delta \nu_{p}=0.3175$.

\section{Doppler Measurements with the Passive Cavity}

Suppose the mode-locked laser is moving toward our receiver with a velocity $v$ as indicated in Fig. 1 . For TEM waves, ${ }^{15}$ an emitted frequency $\omega^{\prime}$ will be observed up-shifted to $\omega$ given by $\omega=\gamma(1+v / c) \omega^{\prime}$ in which $\gamma=\left[1-(v / c)^{2}\right]^{1 / 2}$. Assuming normal incidence, by Eq. (7) the input signal, i.e., the Doppler shifted electric field, is readily expressed as:

$$
\begin{aligned}
E_{1}(\mathrm{t})= & \left(\left\{\sin \left[(2 N+1) \omega_{\mathrm{c}} \gamma(1+v / c) t / 2\right]\right\} /\left\{\operatorname { s i n } \left[\omega_{c} \gamma\right.\right.\right. \\
& \times(1+v / c) t] / 2\}) \cdot \exp \left[i \omega_{0} \gamma(1+v / c) t\right] .
\end{aligned}
$$

Thus, in the case where there is relative motion, optimal detection of the mode-locked laser signal requires a receiver with a cavity length, $h_{o}$, given by

$$
h_{0}=\pi c /\left\{\omega_{c} \gamma[1+(v / c)]\right\}=h /\{\gamma[1+(v / c)]\} \text {. }
$$

Similarly if the mode-locked laser and the passive cavity were on a common platform, the echo from a vehicle moving toward this platform with velocity $v$ would be shifted to $\omega=(1+2 v / c) \omega^{\prime}$, where we have set $\gamma=1$. So by vernier adjustments (PZT driven mirror) of the passive cavity we can read a large range of approach velocities, with a resolution independent of the velocity $v$, i.e. $\delta h / h \approx \delta v / c$ for $v / c \ll$ 1. Thus with $\delta h / h=3.3 \times 10^{-8}$ we find a resolution of $\delta \mathrm{v} \cong 10 \mathrm{~m} / c$.

\section{Experiment}

In this experiment the SNR improvement by predetection filtering of the optical input by a passive Fabry-Perot resonant cavity was measured as a function of several parameters: relative cavity lengths and passive cavity finesse (Fig. 1). Since the passive cavity is a good approximation to the matched-filter for mode-locked lasers only when the cavity lengths are matched, the detector output was monitored for various relative cavity lengths. The mode-locked laser signals were obtained from a self-mode-locking $\mathrm{He}-\mathrm{Ne}$ laser operating at $0.633 \mu \mathrm{m}$ of length $1.2 \mathrm{~m}$ with an average power output of 2 $\mathrm{mW}$. The change in length of the laser $h$ was pro- 


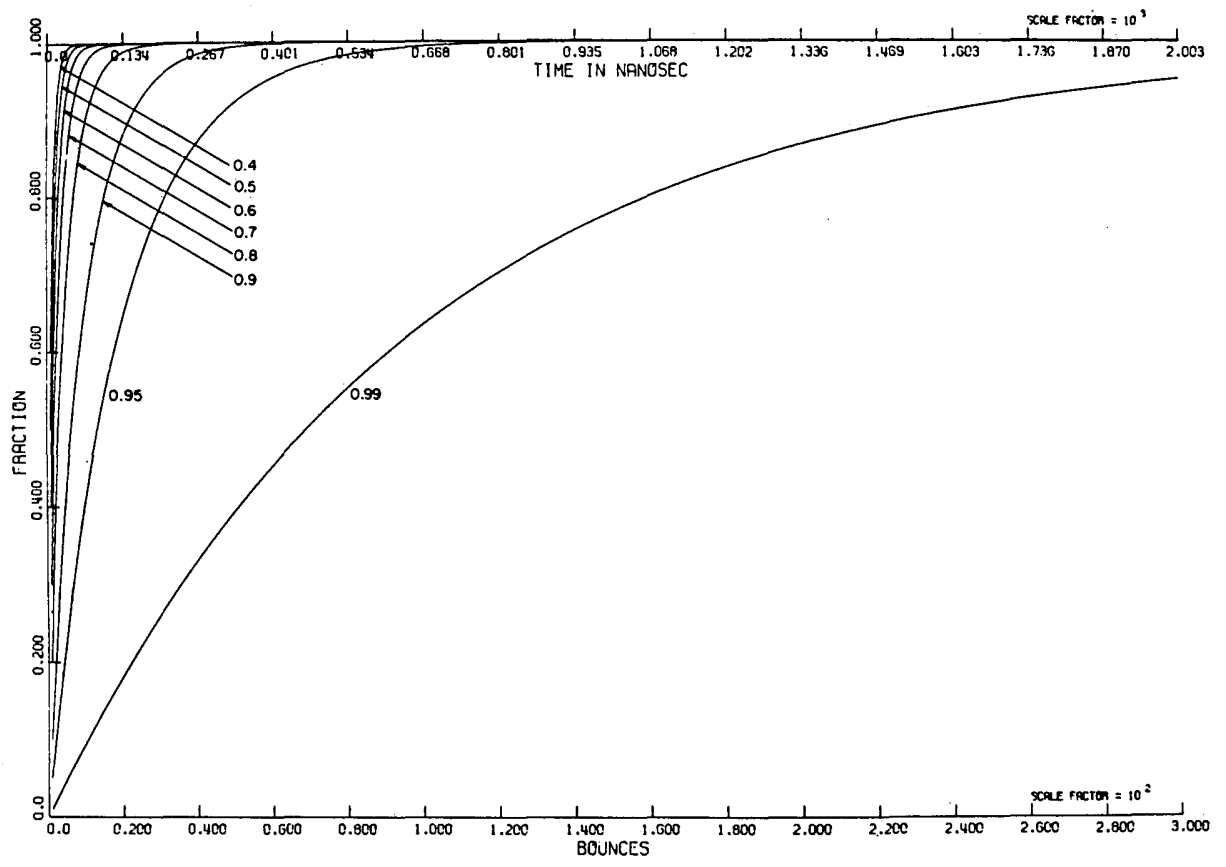

Fig. 4. Fraction of the maximum output vs time in nanoseconds and vs the number of complete bounces $M$, parameterized for various $R_{\alpha}=r_{1} r_{2} \exp (-2 \alpha h)$.

vided by the motion of mirror $M_{3}$ on Teflon runners while the fine fractional wavelength control $(1 \mu \mathrm{m}$ full scale) of the passive cavity length $h_{o}$ was provided by a PZT driven mirror $M_{2}$. The passive cavity was fabricated with the mirrors on Invar rods to reduce thermal variations while the entire experiment was performed in a controlled acoustical environment that provided isolation $(\sim 70 \mathrm{~dB})$ from external turbulence and mechanical fluctuations. The passive cavity was scanned at a $1-\mathrm{Hz}$ rate to eliminate the remaining fluctuations. Thus continuous monitoring of the cavity output, while the length of the laser cavity was changed, allowed us to match exactly the cavity lengths by peaking the output. Note that all the modes of the laser are transmitted simultaneously in the matched condition. The power output was observed to go as approximately $(2 N+1)^{2}$, i.e., as the square of the number of modes oscillating.

To measure the SNR improvement, white noise $N_{o}$ from an ac driven tungsten lamp at $3200 \mathrm{~K}$ was introduced axially into the system. The power from the noise source, passed through a coarse band pass filter, $\mathrm{F}$, at the input of the passive cavity, was 1.5 $\mathrm{W}$ in a $100-\AA$ band around $0.633 \mu \mathrm{m}$. The thermal noise of the TIXL55 avalanche diode detector in a $500-\mathrm{MHz}$ range was far above the shot noise value $\left(2 e I_{o} \Delta f\right)^{1 / 2}$ for operating currents of $0.1 \mu \mathrm{A}$; so, throughout this discussion, we shall be considering the signal-to-unwanted signal ratio. Mirror $M_{1}$ is a standard Spectra-Physics flat laser reflector with reflectivity 0.991. Mirror $M_{2}$ is a standard SpectraPhysics Laser reflector with radius of curvature $2 \mathrm{~m}$ and reflectivity 0.991 . Both reflectivities were chosen to maximize the ideal finesse $\mathbf{F} \equiv \pi \sqrt{ } P / 2$ by minimizing loss. In all cases the measured finesse (245 max) was lower than the ideal (346) due to mirror surface roughness and scattering from occlusions in the mirror multilayer. This factor as well as the finesse was measured by means of a Spectra-Physics Model 119 single mode laser which provided the delta in frequency required to study the spectral response of the cavity.

With the cavity set for the largest Fresnel number (50), i.e., end apertures were limited by the mirror dimensions, the signal and noise were measured with $M_{1}$ and $M_{2}$ aligned to maximize the passive cavity finesse. The finesse was also measured and found to be 245 . The mirrors of the passive cavity were then removed and the signal and noise were measured again. The ratio of $\mathrm{SNR}_{\mathrm{with}}$ to $\mathrm{SNR}_{\mathrm{without}}$ was 156 , and from Eq. (22) we see that the theoretical improvement using the experimentally determined finesse is $2(245) / \pi=156$. The experiment was repeated for various values of finesse and the results are summarized in Fig. 5 along with a plot of Eq.

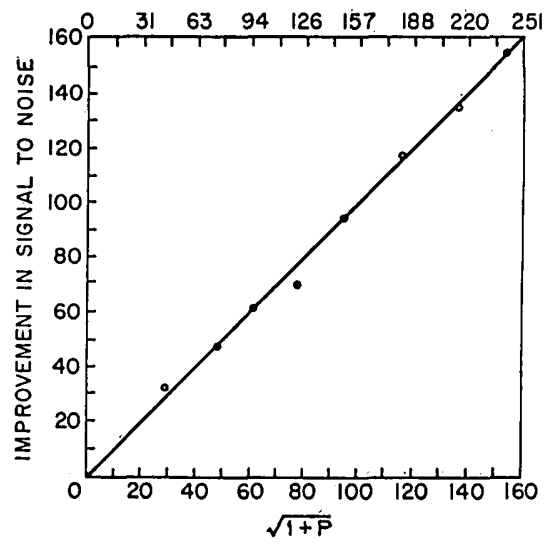

Fig. 5. Signal-to-noise improvement with passive cavity. 
(22). All measurements were made using density filters calibrated at $0.633 \mu \mathrm{m}$ to avoid nonlinearities in the detector electronics.

\section{Summary}

In this work we have shown that the passive Fabry-Perot resonant cavity which is equal in length to the laser cavity is probably as close a physical realization to a matched-filter for multitoned modelocked gas laser signals as can be attained in a passive system. For the passive cavity in contrast to no cavity the SNR improves by the factor $\left(P_{2}+1\right)^{1 / 2}$ which is typically 100-200 for a cavity of good finesse. Also, the peak value of the temporally varying SNR improves as $(2 N+1)^{2}$, i.e., as the peak power of the mode-locked laser.

An alternative approach to the mode-locked laser lineshape is presented in Eq. (10) along with the departure of the passive cavity from the matched-filter for $\Delta \omega_{1}<\Delta \omega_{p}$ and $h \neq h_{o}$. We obtain an expression for the rise-time which is also a function of $\Delta \omega_{p}$ and for cavities of high finesse independent of the number of modes detected.

Further improvement of the SNR above that obtained with the passive cavity may be accomplished with subsequent processing of the detector output, such as boxcar-integration of time-sampled displays. In applications of the receiver to information modulated multitone lasers, the effective bandwidth of the passive Fabry-Perot can be controlled by appropriate choices of $r_{1}$ and $r_{2}$ in Eq. (2).
The authors would like to acknowledge that the research was supported in part by the Air Force Office of Scientific Research and to thank Bell Telephone Laboratories for their doctoral support fellowship during the completion of this study.

\section{References}

1. L. E. Hargrove, R. L. Fork, and M. A. Pollack, Appl. Phys. Lett. 5, 4 (1964).

2. W. H. Steier, Proc. IEEE 54, 1604 (1966).

3. R. L. Fork, D. R. Herriott, and H. Kogelnik, Appl. Opt. 3, 1471 (1964)

4. H. Kogelnik and A. Yariv, Proc. IEEE 52, 165 (1964).

5. R. W. Uhlhorn and D. F. Holshouser, IEEE J. Quantum Electron. QE-6, 775 (1970).

6. C. M. Sonnenschien and F. A. Horrigan, Appl. Opt. 10, 1600 (1971).

7. M. J. Rudd, J. Phys. E 2, 55 (1969).

8. Equation (1) is a generalization, to include frequency variations of $t_{1}, t_{2}, r_{1}$, and $r_{2}$ of the well known expression for cavity transmissivity in a Fabry-Perot, e.g., M. Born and E. Wolf, Principles of Optics (Pergamon Press, Oxford, 1970), pp. $62,327$.

9. W. M. Brown, Analysis of Linear Time-Invariant Systems (McGraw-Hill, New York, 1963), p. 245.

10. A. Blaquiere, Ann. Radioelectr. 8, 36 (1953).

11. J. P. Gordon, H. J. Ziegler, and C. H. Townes, Phys. Rev. 99, 1264 (1955).

12. C. Freed and H. A. Haus, Appl. Phys. Lett. 6, 85, (1965).

13. P. Grivet and A. Blaquiere, in Symposium on Optical Masers, J. Fox, Ed. (Polytechnic Press, Brooklyn, 1963), p. 69.

14. A. Yariv, Quantum Electronics (John Wiley, New York, 1967), p. 409.

15. C. H. Papas, Theory of Electromagnetic Wave Propagation (McGraw-Hill, New York, 1965). p. 225.

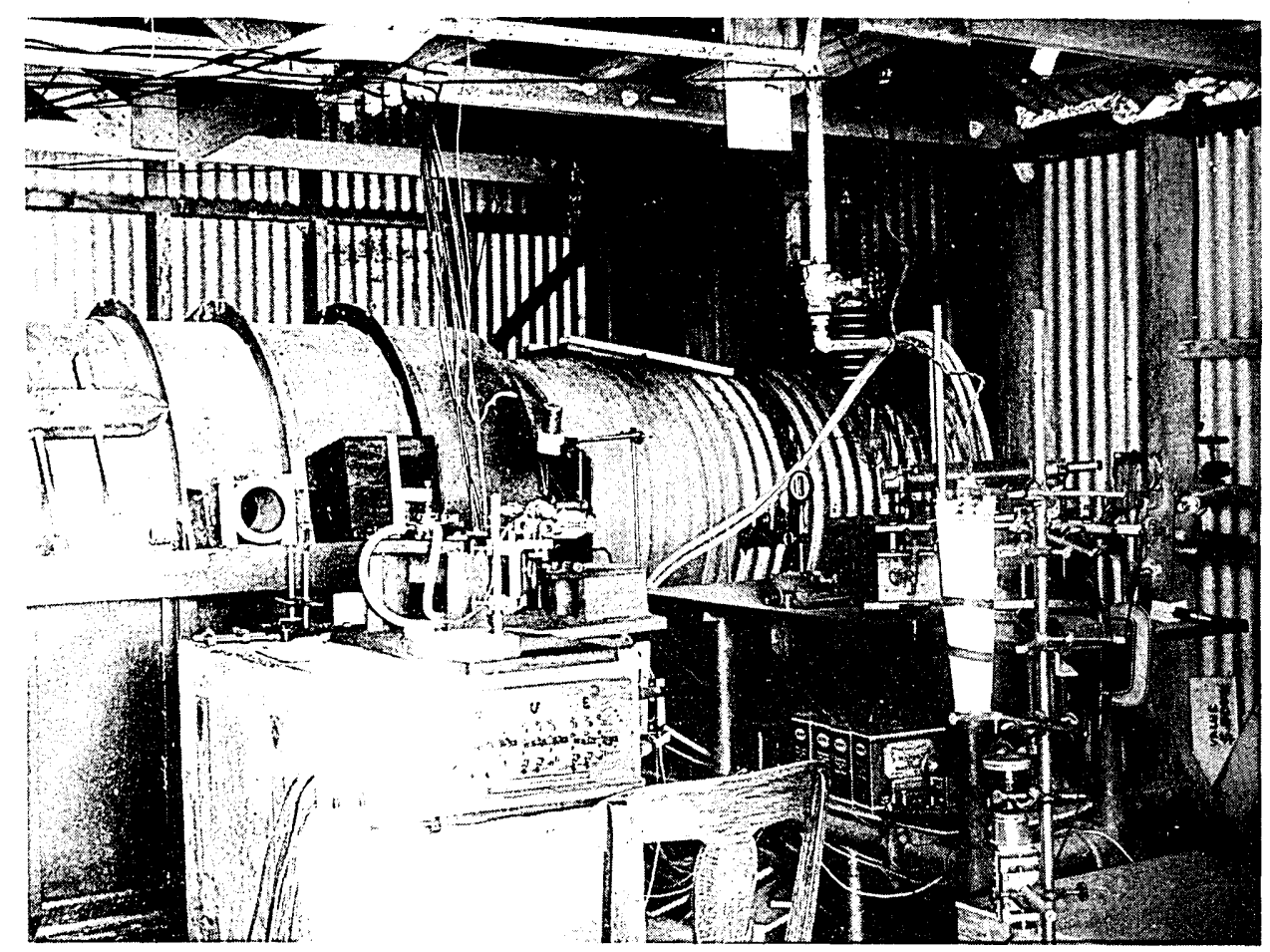

Interior of the shed at the Irvine Ranch housing the rotating mirror, telescope, etc. used in the experiment to measure the velocity of light in vacuum. (From the Michelson Museum collection) 